\title{
Varios presentes, varios futuros: consideraciones para la educación en lo incierto
}

\author{
doi: $10.52749 /$ fh.v2i4.6
}

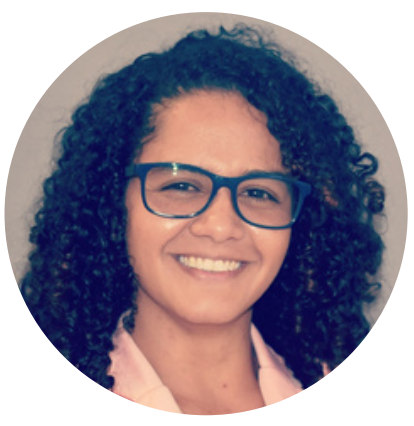

\begin{abstract}
MARCELA GÓMEZ
Fundadora del Colectivo Tejido STEM Colombia. Miembro del Colectivo Nacional de Educación STEM - CONASTEM - y del Colectivo Latinoamericano Manifiesto STEAM. Coordinadora STEM del Colegio Colombo Hebreo (Colombia). Miembro asesor de la Mesa Técnica Nacional de Educación STEM+A para el Ministerio de Educación Nacional de Colombia. Ingeniera Mecánica y Magíster en Ciencias Naturales y Matemática de la Universidad Pontificia Bolivariana (Colombia). Diplomada en Educación STEM/STEAM por la OEA. STEM.org Certified Educational Instructor.
\end{abstract}

marcelagq1987@gmail.com

O० @marcela.stem

Resumen. Este artículo busca presentar al lector una revisión de las características que pueden influir significativamente en la consolidación de una mentalidad orientada al aprendizaje en lo incierto. Para tal propósito, se brinda una descripción breve de la dinámica social actual, la cual condiciona el ejercicio educativo y formativo, y después de proponer una lectura tripartita de los elementos involucrados en la definición de un comportamiento armónico de la comunidad a partir del modelo de Swift en Los viajes de Gulliver. Gracias a los perfiles presentados por este texto (aprendiz, mentor y espectador), se sugieren algunas características necesarias en la educación para que permita la creación de una mentalidad sólida ante la incertidumbre, que incluyen entre otras, la motivación intrínseca, la mentoría líquida, y la documentación de experiencias.

Palabras clave: educación, dinámica de sistemas, procesos de pensamiento, cultura comunitaria.

"[...]Su Majestad, [...] Me inquirió sobre los métodos empleados en la educación física e intelectual de nuestros jóvenes patricios, y en qué se ocupaban durante los años de formación, especialmente en los primeros años de su vida, más aptos para ser instruidos." (Swift, 1726, p. 163)

Una mirada fugaz a nuestro comportamiento cotidiano permite afirmar que la sociedad actual se caracteriza por la rápida (y superficial) interacción de un individuo con sus alrededores y sus actores, es decir, que ha desarrollado unos hábitos de selección exprés de datos de cara a la construcción de información que permita, de igual manera, construir más rápidamente un mundo acorde con la increíble cantidad de estos datos disponibles en cada vez menos tiempo. Para facilitar la carga cognitiva, esta sociedad cuenta con modelos visuales y considerablemente filtrados de un estilo de vida unidireccional y pasivo [1], los cuales condicionan en gran medida las aplicaciones de las herramientas (cada vez más digitales) que en un principio buscan responder a una mayor calidad de vida para la mayor cantidad de personas (Pinker, 2018). También es posible sugerir que esta sociedad ha hecho más explícito un sentido de comunidad universal, cercano en muchos casos a lo tribal, en donde hay una defensa férrea del otro ante posibles situaciones que se interpretan como atentados al reconocimiento de su individualidad. Puede hablarse de una percepción dual de la cotidianidad humana, en la que dos aspectos aparentemente contradictorios - la prevalencia del individuo y la pertenencia (al menos nominal) a una comunidad establecen una relación que podría catalogarse como mutualista.

Teniendo en cuenta la vigencia de esta percepción individual-comunitaria de la realidad, se reconoce un entramado de circunstancias históricas que tienen un impacto en la interpretación que cada individuo hace de la misma en un instante determinado, y que consolidan el modelo construido para responder a las demandas de su respectiva cotidianidad; esto concuerda con la afirmación popular de que "hay tantos mundos como personas (que) lo habitan". Entonces, los datos procesados por cada persona en el marco de su vivencia cotidiana refuerzan o modifican el modelo individual creado para predecir su futuro próximo y, por qué no, mediato. Dichos datos, a su vez, son recolectados e interpretados por otras personas según la estructura de sus propios mode- 
modelos, creando respuestas que pueden diferir más o menos de las de sus cohabitantes. Desde esta perspectiva, existen realidades simultáneas que constan de hechos e interpretaciones; mientras los primeros son irrefutables, las segundas son procesadas por los modelos mentales de cada individuo, los cuales son a su vez influidos por los órdenes imaginados establecidos en la comunidad a la cual pertenece (Harari, 2015).

Ahora, ¿cuál de todas es la más cercana a lo que podría pasar en un futuro? ¿Qué tal si nueva información (datos recolectados y procesados bajo un modelo cualquiera) es simplemente difundida, se integra a esta nube y altera drásticamente el curso de un hito en la Historia? Sin afán de promover el fatalismo, este artículo sostiene que la conciencia de las realidades simultáneas permite reconocer a la incertidumbre como factor cierto en la dinámica de este sistema humano $y$, por tanto, lo posiciona como aspecto de gran relevancia en la construcción de mentalidades individuales y colectivas, presentes y futuras.

El ejercicio de las realidades simultáneas no es nuevo, a decir verdad, sino que ocupa escenarios diversos relacionados con la toma de decisiones (Amster \& Pinasco, 2009), la comunicación holística [2] Llamas Álvarez, 2021) y la literatura. En este último escenario, un ejemplo brillante es el de Los viajes de Gulliver, libro escrito por el irlandés Jonathan Swift en 1726 y que hace una lectura afilada de las estructuras sociales de su país (así como de sí mismo) a través de los ojos de distintos interlocutores entre los que se incluyen los liliputienses, los brobdingnagianos, los houyhnhnms y los yahoos [3]. Al leer las peripecias de Gulliver en su trasegar por el mundo, se evidencian los siguientes patrones:

- Cada nuevo territorio ve su extensión como la extensión del mundo, incluso del Universo. Una vez Gulliver llega, este modelo se tambalea.

- El acercamiento a lo desconocido (es decir, a Gulliver) por parte de la comunidad estuvo condicionado por la respuesta de un líder comunitario. Este líder muestra (o debería mostrar) las virtudes de la comunidad en pleno.

- En tanto hubo una mayor familiaridad con lo inicialmente desconocido, se hicieron visibles las respuestas éticas y morales propias de la comunidad.

Según lo anterior, hay una ruta definida para la interacción de Gulliver con cada reino nuevo al que llega, la cual no garantiza resultados unánimes, ni siquiera cercanos entre sí. Puede considerarse esto como una invitación a observar el mundo y a sus habitantes estocástica y continuamente: considerando los momentos históricos como diferenciales de tiempo, y asumiendo sus respuestas probabilísticamente. Desde luego, la complejidad de este panorama puede abrumar al lector, así que, en procura de reducir la carga cognitiva, se proponen tres miradas básicas para la construcción de un modelo medianamente holístico que propende por la educación en lo incierto: la mirada del aprendiz, la mirada del mentor, y la mirada del espectador.

La mirada del aprendiz (representado por Lemuel Gulliver o el viajero)

"Si quieres construir un barco, no empieces por buscar madera, cortar tablas o distribuir el trabajo. Evoca primero en los hombres y mujeres el anhelo del mar libre y ancho". Antoine de Saint-Exupéry

El viajero se mueve motivado por su afán de navegar. Más allá de sus necesidades económicas, sociales, políticas y demás, su interés por explorar y conocer nuevas tierras constituye el motor de sus decisiones. Esto indica que existe en él una fuerte motivación intrínseca que lo impulsa a adentrarse en lo desconocido. ¿De qué depende esta motivación intrínseca? Según el texto, la inmersión en escenarios de aprendizaje ricos y diversos que implican no solamente lo académico, sino también la interacción con oficios y trabajos manuales, hicieron mella en el personaje (Dehaene, 2018), generando en él hábitos de interacción directa con su entorno desde un aprendizaje gradual, en donde fue siempre el primer responsable de su aprendizaje (Gómez Quintero, 2019, 2021).

Desde lo anterior también hay que mencionar que, para hacerse a la mar, debió garantizar una serie de habilidades que le permitieran asumir un rol en cada embarcación, así como otras más específicas que le posicionan como alguien valioso para cada misión. Esto quiere decir que, independientemente de cada rol, hay aspectos básicos e innegociables que deben ser conocidos por todos para la adecuada operación del barco bajo cualquier circunstancia. Se presenta de manera implícita una estructura preparada para enfrentarse a situaciones probables variadas, no garantizadas, pero sí fundamentadas en datos históricos, así como en modelos creados a partir de los mismos. Esto permite, hasta cierto punto, configurar el entorno cercano para hacerle interactuar de manera controlada con lo inesperado, o por lo menos con lo 
hasta ahora incontrolable, pudiendo dar respuesta a múltiples escenarios probables (Oppenheimer, 2014; Latorre, 2019).

Finalmente, pero no menos importante, el viajero pasó por experiencias diversas y no necesariamente placenteras, que no menguaron en absoluto su deseo de seguir viajando. Su mente reconoce las experiencias como parte del proceso de aprendizaje, como iteraciones que refinan sus modelos mentales en torno al mundo que explora (Botero, 2018; Dehaene, 2018), y que permiten obtener o crear herramientas para futuros retos. Sin duda, este aprendiz cuenta con una mentalidad proactiva que le permite percibirse como ser influyente en un futuro que aún no llega (Gómez Quintero, 2021).

La mirada del mentor (personificado por cada uno de sus interlocutores en las tierras visitadas a lo largo de sus viajes; cada uno puede ser llamado anfitrión)

"El maestro deja una huella para la eternidad; nunca puede decir cuándo se detiene su influencia".

Henry Adams

Desde sus dominios, el mentor recibe al aprendiz sin esperarlo. Esto deja claro que este mentor es también un aprendiz, un aprendiz de quien llega a sus terrenos. La primera respuesta de cada anfitrión ante este sorpresivo visitante no solamente brinda pistas respecto de cómo puede evolucionar la relación posterior, sino que además permite rumorar alrededor de cómo se ve a sí mismo cuando se enfrenta a lo desconocido. Ciertamente, Lemuel Gulliver notó cada uno de estos detalles, pues los describió en sus relatos.

En efecto, las experiencias de recibimiento en cada territorio fueron diferentes a pesar de contar con varios elementos en común:

- Los liliputienses percibieron a Gulliver como una amenaza, principalmente por su gran tamaño. En principio, no prestaron atención suficiente al hecho de que era físicamente semejante a ellos, siendo sus dimensiones la única diferencia esencial; más adelante, esta particularidad fue tenida en cuenta para el cálculo de recursos necesarios en su mantenimiento, del mismo modo que se consideró en términos de los beneficios que podría traer a Liliput en su conflicto con Blefuscu, la isla vecina.
- Los brobdingnagianos observaron a Gulliver con curiosidad, incluso con condescendencia y algo de diversión. Lo utilizaron como diversión para ganar dinero en ferias y otros eventos, incluso a costa de su salud, y sólo fue tratado de manera más digna en presencia de los monarcas; allí tuvo la oportunidad de poner a prueba sus principios en amplios debates donde fue escuchado atentamente y confrontado con preguntas basadas en sus propios testimonios, las cuales fueron formuladas desde la genuina curiosidad de su actual benefactor, el rey.

- Los houyhnhnms, si bien también se mostraron asombrados ante la presencia de Gulliver, mostraron una actitud más científica: no tuvieron prisa en reaccionar, sino que se tomaron el tiempo para observar, reflexionar y discutir con sus pares, mientras mantenían un contacto mesurado con él hasta encontrar la mejor manera de interactuar. Es necesario considerar que éstos son, de los anfitriones aquí presentados, los que exhiben mayores diferencias físicas, por lo que inicialmente se habría esperado un primer encuentro chocante.

- Los yahoos, por último, fueron significativos por su comportamiento bestial en contraste con su apariencia humanoide. Su contacto con Gulliver no fue tan parecido a los anteriores, pero tuvo un fuerte impacto indirecto: su manera de gruñir, de moverse, de comer, y en general de vivir, hizo que Gulliver sintiera una poderosa aversión desde un primer momento, aversión que perduró incluso hasta mucho después de regresar a su tierra natal. Sus acciones cotidianas fueron suficientes para afectar a Gulliver en lo profundo.

Todo este abanico ayuda a establecer un mapa de comportamientos y hábitos que influye en la respuesta del aprendiz desde el primer momento. Es importante resaltar que este mapa fue significativamente trazado por cada uno de los líderes anfitriones, pero que fue resaltado o emborronado por todos aquellos individuos de los reinos visitados, ya fueran miembros de la corte o simples plebeyos, ciudadanos comunes, nobles o prisioneros. Así, el carácter líquido del rol de mentoría se hace plausible, y su influencia se materializa en toda la extensión de las memorias de Gulliver, más allá de las modificaciones hechas por su editor. 
La mirada del espectador o lector

"En este teatro de la vida del hombre, está reservado sólo a Dios y a los ángeles ser espectadores". Francis Bacon

Ahora, es el turno de quien ha leído las crónicas. No solamente de quien las leyó en su primera impresión, sino de quien las lee casi trescientos años después (incluyéndome). Quien ha leído el libro ha podido relacionar elementos de la fisionomía, los comportamientos y las decisiones de los personajes con sus vivencias propias y algunos testimonios ajenos (pasados por sus modelos de la realidad, claro) y, en función de la profundidad con la que suele aproximarse al conocimiento por estos medios, ha podido establecer una relación metatextual con Swift a través de Gulliver (De Zubiría Samper, 1996).

A pesar de la distancia espacio-temporal, es posible reconocer la vigencia del texto. No obstante, los niveles de profundidad que se pueden intuir en el marco de esta vigencia dependen en gran medida de la cantidad de conexiones que cada lector pueda hacer con el saber que ha construido a lo largo de su vida. Entre más conexiones se den, más caminos podrán crearse entre una idea y otras tantas para hacer del modelo individual de la realidad una herramienta poderosa de transformación mediante la identificación de más escenarios posibles. Entre más escenarios, mayor influencia tiene cada lector en la materialización del porvenir.

\section{Conclusión}

En suma, la incertidumbre es una característica innegable de nuestra contemporaneidad, ya que la cantidad de datos que son consumidos y modificados día a día tienen un impacto considerable en las decisiones hacia el futuro; con todo y las actuales metodologías, técnicas y herramientas para el procesamiento de datos, el peso de la inercia individual cotidiana sigue actuando como un ancla que reduce nuestro movimiento en este océano. Entonces, una revisión de cómo puede observarse el panorama vigente, desde las miradas de los distintos tripulantes del barco, puede ayudar en el proceso de ajustar el rumbo y finalmente elevar anclas. Pueden mencionarse los siguientes aspectos como clave para la educación en lo incierto:

- Interacción directa y visible con el entorno, con orientación de un mentor.
- Actitud de observación cuidadosa y profunda ante lo desconocido.

- Genuina curiosidad, manifiesta a través de preguntas investigables y de confrontación.

- Pensamiento operativo, en cuestión de los recursos necesarios para interactuar de la mejor forma posible con nuestro sujeto/objeto de interés.

- Conciencia del impacto de las acciones cotidianas en la respuesta de los actores alrededor.

- Hábitos de lectura, procesamiento y evaluación de datos en el proceso de construcción de conocimiento.

- Conciencia de la relevancia de la historia en la construcción de un presente y un futuro.

[1] Esto se refiere a un estilo de vida en donde los bienes fluyen en una sola dirección (hacia el usuario) haciendo el mínimo esfuerzo posible.

[2] Entendida desde la coinfluencia entre sistemas complejos en permanente interacción. El autor se refiere a una comunicación correspondiente $y$ corresponsable, en la cual un sujeto interactúa con otro sujeto (o con un objeto) y con sus circunstancias.

[3] Cada uno de estos personajes (y por extensión, sus comunidades) ofrece una personificación de las características del ser humano, tanto en sus virtudes como en sus vicios.

\section{Referencias}

Amster, P. \& Pinasco, J. P. (2009). Teoría de juegos. Una introducción matemática a la toma de decisiones. Fondo de Cultura Económica.

Botero, J. (2018). Educación STEM. Introducción a una nueva forma de enseñar y aprender. STEM Education Colombia.

De Zubiría Samper, J.(1996). Teoría de las Seis Lecturas. Fundación Alberto Merani.

Dehaene, S. (2018). En busca de la mente. Siglo Veintiuno Editores Argentina.

Gómez Quintero, L. M. (2019) Educación STEM/STEAM como pretexto para la innovación en comunidades de aprendizaje. En N M. Cáceres (Ed.), Educación STEM / STEAM, apuestas hacia la formación, impacto y proyección de seres críticos (p. 56). Fondo Editorial Universitario Servando Garcés de la Universidad Politécnica Territorial de Falcón.

Gómez, M. (2021). Cultura para la transformación vs. la mentalidad de superviviente. Futuro Hoy, 2(1), 13-15.

https://doi.org/10.52749/fh.v2i1.4

Harari, Y. N. (2015). Homo Deus. Penguin Random House Latorre, J. I. (2019) Ética para máquinas. Editorial Planeta Colombiana. 
Llamas Álvarez, J. T. (2021). La comunicación holística como método de vida. Impulso Gráfico Ltda.

Oppenheimer, A. (2014) ¡Crear o morir! La esperanza de América Latina y las cinco claves de la innovación. Penguin Random House Grupo Editorial.

Pinker, S. (2018). En defensa de la ilustración. Paidós Casa Editorial. Swift, J. (1726). Los viajes de Gulliver. Penguin Random House Grupo Editorial.

\section{Cómo citar este artículo:}

Gómez, M. (2021). Varios presentes, varios futuros: consideraciones para la educación en lo incierto. Futuro Hoy, 2(4), 31-35. https://doi.org/10.52749/fh.v2i4.6

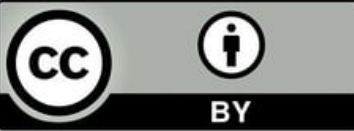

Esta obra está bajo licencia internacional Creative Commons 4.0 Reconocimiento 4.0. 